\title{
A Tailgut Cyst-Cystic Mass Diagnosed by Prenatal Ultrasonography
}

\author{
Ki Yeong Chung, MB ${ }^{1}$ Na Mi Lee, MD ${ }^{1}$ Eung Sang Choi, MD, PhD ${ }^{1}$ Byoung Hoon Yoo, MD, PhD \\ Gwang Jun Kim, MD, PhD ${ }^{2}$ Seong Jae Cha, MD, PhD ${ }^{3}$ Gi Hyeon Kim, MD, PhD ${ }^{4}$ Mi Kyung Kim, MD, PhD
}

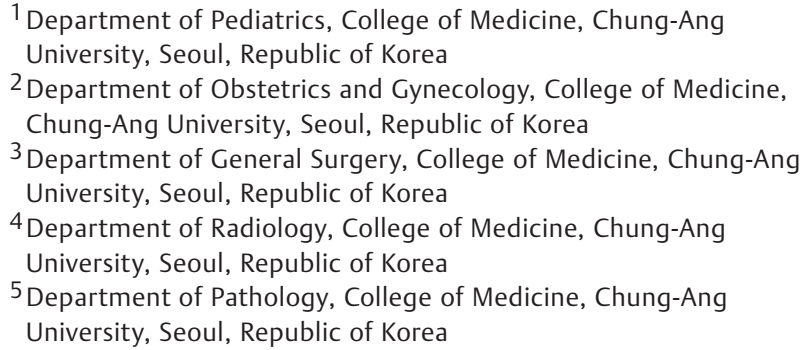

Address for correspondence Na Mi Lee, MD, Department of Pediatrics, College of Medicine, Chung-Ang University 224-1 Heukseok-dong, Dongjak-gu, Seoul, Republic of Korea (e-mail: piena81@hotmail.com).

Am J Perinatol Rep 2013;3:17-20.

\begin{abstract}
Keywords

- tailgut cyst

- neonate

- prenatal ultrasonography

Tailgut cysts are congenital lesions found in the presacral space. These have been mainly identified in adults and are rare in children, especially neonates. Here, we present the case of a neonate with a presacral cystic mass detected by prenatal ultrasonography that was diagnosed as a tailgut cyst after postnatal surgical removal. When a presacral cyst is encountered, tailgut cyst should be considered in the differential diagnosis.
\end{abstract}

The tailgut cyst, also known as retrorectal cystic hamartoma or mucus-secreting cyst, is a congenital lesion found in the presacral space. $^{1,2}$ It arises from vestiges of the embryonic hindgut and is characterized by multiple cysts lined with a gastrointestinal type of epithelium. ${ }^{3}$ This type of lesion has been mainly described in adults and rarely in children, especially neonates. Here, we report a case of a neonate with a presacral cystic mass detected on prenatal ultrasonography that was confirmed to be a tailgut cyst after postnatal surgical removal.

\section{Case Report}

A 31-year-old, gravida 2, para 1 pregnant woman was referred to our hospital due to a presacral cyst that had appeared on a prenatal ultrasonogram. The medical history, physical examination results, and family history were unremarkable. Detailed ultrasonography showed a female fetus approximately 25 weeks of age according to biometric parameters; this was consistent with the estimated date of delivery.
A bilocular cyst (longest diameter, $1.5 \mathrm{~cm}$ ) was detected in the presacral region (-Fig. 1). The lumbar, sacrum, and coccyx were intact, and no abnormalities were found in the brain. During the follow-up period, the size of the cyst increased to $2.7 \mathrm{~cm}$ at 29 weeks and $4.5 \mathrm{~cm}$ at 36 weeks of gestation.

A 3.81-kg baby girl was born at 40 weeks, 2 days of gestation by normal spontaneous vaginal delivery. After birth, she was found to be healthy as included by an Apgar score of 9 at 1 minute, and 9 at 5 minutes. Upon a physical examination, a soft, bluish, round mass was found at sacrococcygeal area. The mass was a $4 \times 5 \mathrm{~cm}$ in size at the midline, was not mobile, and had no skin defects.

On day 2 of hospitalization, coccygeal ultrasonography was performed. Longitudinal ultrasonogram with a highfrequency transducer showed the pure cystic masses around the coccyx (-Fig. 2A). On day 3 of hospitalization, pelvis magnetic resonance imaging (MRI) was performed. The T2-weighted images along the midsagittal and axial planes depicted the pure cystic masses more clearly with high signal intensity both anterior and posterior to the $\operatorname{coccyx}$ (-Fig. 2B). received

January 9, 2012

accepted after revision

July 25,2012

published online

December 3, 2012
Copyright $\odot 2013$ by Thieme Medical Publishers, Inc., 333 Seventh Avenue, New York, NY 10001, USA. Tel: +1(212) 584-4662.
DOI http://dx.doi.org/ 10.1055/s-0032-1329681. ISSN 2157-6998. 


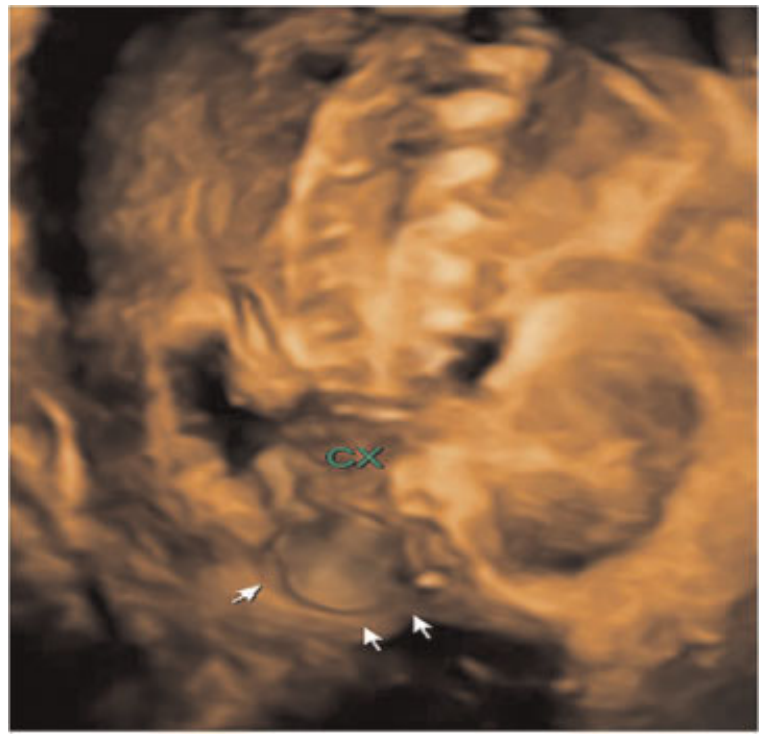

Fig. 1 Small coccygeal cyst apparent on prenatal three-dimensional ultrasonography at gestational week 25 . Arrows indicate the small coccygeal cyst with an intact sacrococcygeal architecture. CX, coccygeal bone.

T1-weighted axial MRI after gadolinium injection revealed cystic masses with low signal intensity and thin wall enhancement. There was no evidence of local invasion or bony destruction. Thus meningocele can be ruled out. The serum $\alpha-$ fetoprotein level was $>60,500 \mathrm{ng} / \mathrm{mL}$ (normal range, $33,113 \pm 32,503 \mathrm{ng} / \mathrm{mL})^{4}$

On day 9 of hospitalization, the cystic mass was surgically removed. The patient was operated on under general anesthesia and in the prone jackknife position. A skin incision of $3 \times 3 \mathrm{~cm}$ was made in an inverted $V$ shape on the lesion. The mass was found to be a multiloculated cyst adhering to the sacrum bone and extending in presacral area. The mass was carefully dissected from the posterior wall of the rectum and removed with the coccyx. The microscopic characteristics of the cystic mass were consistent with those of a tailgut cyst (-Fig. 3).

After the operation, the patient's vital signs were stable. At the age of 14 days, the serum $\alpha$-fetoprotein level had decreased to $7675 \mathrm{ng} / \mathrm{mL}$ (normal range, $9452 \pm 12,610$ ). ${ }^{4}$ The drop of $\alpha$-fetoprotein level means clear mass resection without meningeal invasion. The patient fully recovered and was eventually discharged without developing any sequelae by day 19 of hospitalization.

\section{Discussion}

When a presacral cyst is found by prenatal sonography, obstetricians usually consider some possible diagnoses: sacral meningocele, sacrococcygeal teratoma, or rectal duplication. Bony defects are usually observed, and at least one of five intracranial sonographic signs ${ }^{5}$ can be found in over $99 \%$ of open-type sacral meningocele. ${ }^{6}$ After meticulously searching for sacral defects and intracranial abnormalities with the aid of a three-dimensional ultrasonography skeletal view, we could not find skeletal defects in sacrococcygeal region or intracranial abnormalities in our patient. However, a cyst can be the only evidence of a closed-type neural tube defect. Rectal duplication cysts can be shown spherically or tubularly and are located typically on the dorsal side of the native bowel at prenatal sonography. ${ }^{7}$ But the cyst of our patient was located at the presacral area and had septum between rectum and itself. So the cyst was less likely to be rectal duplication.

Because the baby had a mass at the presacral area, we considered Currarino syndrome, which is a triad of presacral mass, anorectal malformation, and sacral anomaly. ${ }^{8}$ But our case had no anorectal malformation and sacral anomaly, as proven by MRI. Presacral mass can be a meningocele, a teratoma, an enteric cyst, or a combination of these. Anorectal malformation is congenital anorectal stenosis or another type of low anorectal malformation. A sacral defect may be seen as a crescent-shaped deformity of the sacrum in anterior sacral meningocele.

Although only a cystic echo can be visible in some types of cystic teratomas on prenatal sonograms, fetal sacrococcygeal teratomas commonly consist of mixed cystic and solid components and may contain fat or calcified components. Because a bilocular cyst on the sacrococcygeal region was the only sonographic finding in the present case, we could not make a definite diagnosis until birth.

Tailgut cysts are remnants of the embryonic portion of the gut extending beyond the anus. During the course of normal
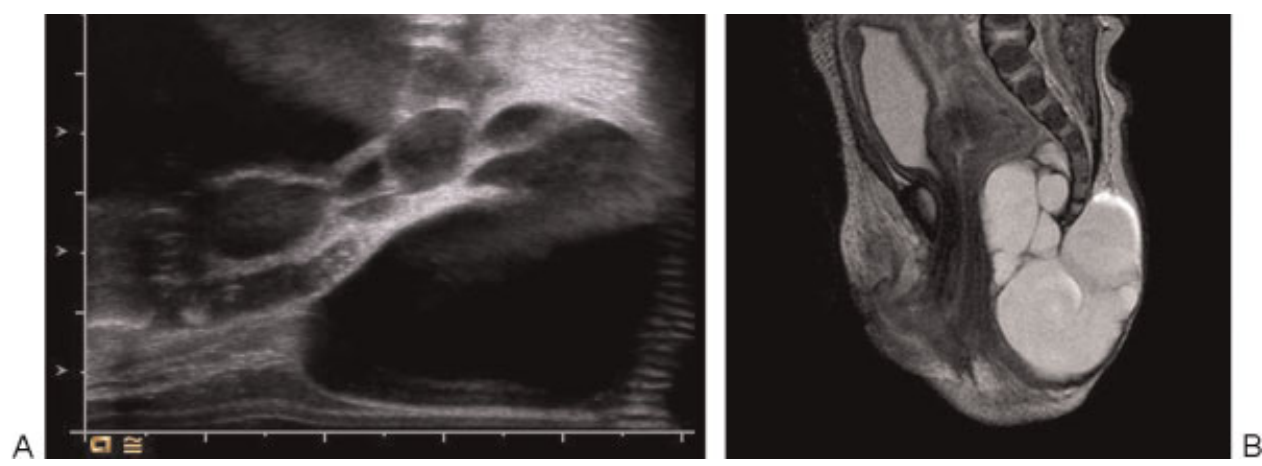

Fig. 2 Radiological findings. Longitudinal ultrasonogram with a high-frequency transducer showed the pure cystic masses around the coccyx (A). T2-weighted magnetic resonance image along the midsagittal and axial planes depicted the pure cystic masses more clearly with high signal intensity both anterior and posterior to the coccyx (B). 


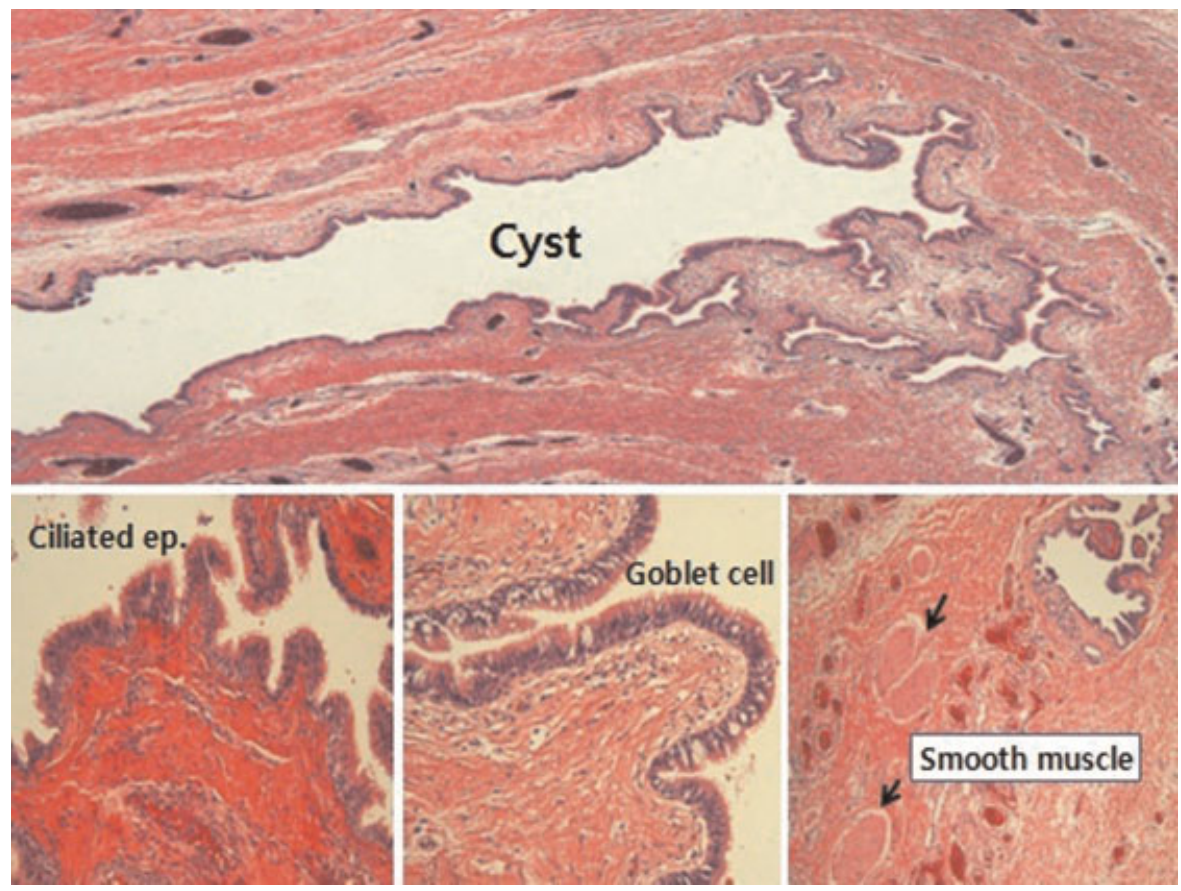

Fig. 3 The cystic wall was lined with epithelial cells and was composed of ciliated and goblet cells. There was also a smooth muscle component within the lesion.

development, the embryo forms a true tail at approximately 35 days of gestation (8-mm stage). The embryonic hindgut extends into the tail, constituting the tailgut or postanal gut, and undergoes regression by gestational age of 56 days. Presumably, the tailgut may persist and lead to the development of tailgut cysts in the retrorectal or presacral spaces. However, Jang et al reported a case of tailgut cysts arising in the prerectal and retrovesical spaces. ${ }^{9}$

The rarity of these tumors and their nonspecific clinical presentations often lead to misdiagnoses. There are several cases in which the initial diagnosis differed from the postoperative diagnosis. ${ }^{10,11}$ Initial suspected diagnoses included perianal abscess, fistula in ano, posttraumatic pain, pilonidal cyst, presacral abscess, postpartum pain, and psychogenic pain. All of these can be differentiated from dermoid cysts by the lack of dermal appendages, from enteric cysts by the lack of intestinal epithelium, and from teratomas that contain all three germ layers. ${ }^{12}$ In our case, we could not make a definite diagnosis until the mass was surgically removed postnatally.

Most tailgut cysts are asymptomatic and are often discovered incidentally. These lesions can also present as compression within the pelvis associated with symptoms such as changes in stool caliber, identification of a palpable rectal mass, changes in urinary frequency, and, frequently, as an infected tailgut cyst with rectal fistula or anal fistula. The most common symptom is pain, especially if the cyst is infected. ${ }^{11}$

Although these cysts can be found in all age groups including infants, they are most prevalent in middle-aged women. ${ }^{13}$ Few cases have been reported in children. Among these, neonatal cases are extremely rare. Hjermstad and Helwig ${ }^{12}$ showed that, among 53 patients with tailgut cysts, 41 were female adults and only one patient (a male) was young ( 1 month of age). There have been five reports of neonatal cases in the literature. ${ }^{13-17}$ Among these previous cases of neonatal tailgut cysts, three cases were identified postnatally and the other two cases were identified prenatally. After the introduction of prenatal ultrasonography, the frequency of intrauterine diagnosis of this lesion has increased. A previous report describing tailgut cysts in neonates and young children also described microscopic and image findings similar to those of our case.

Prenatal MRI can be an option when diagnosing these cases. However, MRI findings cannot change the prenatal management course after 24 gestational weeks, which is generally regarded as the deadline for performing an optional pregnancy termination in South Korea. Furthermore, its high cost can present another potential obstacle.

Tailgut cyst as well as teratoma can rarely become malignant transformations such as adenocarcinoma and carcinoid. ${ }^{18}$ For this reason, this case highlights the need to consider tailgut cyst in the differential diagnosis when a presacral cyst is encountered.

\section{References}

1 Caropreso PR, Wengert PA Jr, Milford HE. Tailgut cyst-a rare retrorectal tumor: report of a case and review. Dis Colon Rectum 1975;18:597-600

2 Rafindadi AH, Shehu SM, Ameh EA. Retrorectal cystic harmatoma (tailgut cyst) in an infant: case report. East Afr Med J 1998;75: 726-727

3 Johnson AR, Ros PR, Hjermstad BM. Tailgut cyst: diagnosis with CT and sonography. AJR Am J Roentgenol 1986;147:1309-1311

4 Wu JT, Book L, Sudar K. Serum alpha fetoprotein (AFP) levels in normal infants. Pediatr Res 1981;15:50-52 
5 Nicolaides KH, Campbell S, Gabbe SG, Guidetti R. Ultrasound screening for spina bifida: cranial and cerebellar signs. Lancet 1986;2:72-74

6 Watson WJ, Chescheir NC, Katz VL, Seeds JW. The role of ultrasound in evaluation of patients with elevated maternal serum alpha-fetoprotein: a review. Obstet Gynecol 1991;78:123-128

7 Haratz-Rubinstein N, Sherer DM. Prenatal sonographic findings of congenital duplication of the cecum. Obstet Gynecol 2003; 101(5 Pt 2):1085-1087

8 Currarino G, Coln D, Votteler T. Triad of anorectal, sacral, and presacral anomalies. AJR Am J Roentgenol 1981;137:395-398

9 Jang SH, Jang KS, Song YS, et al. Unusual prerectal location of a tailgut cyst: a case report. World J Gastroenterol 2006;12:5081-5083

10 Galluzzo ML, Bailez M, Reusmann A, Gonzalez R, de Dávila MT. Tailgut cyst (retrorectal hamartoma): report of a pediatric case. Pediatr Dev Pathol 2007;10:325-327

11 Singer MA, Cintron JR, Martz JE, Schoetz DJ, Abcarian H. Retrorectal cyst: a rare tumor frequently misdiagnosed. J Am Coll Surg 2003; 196:880-886
12 Hjermstad BM, Helwig EB. Tailgut cysts. Report of 53 cases. Am J Clin Pathol 1988;89:139-147

13 Antao B, Lee AC, Gannon C, Arthur R, Sugarman ID. Tailgut cyst in a neonate with anal stenosis. Eur J Pediatr Surg 2004;14: 212-214

14 Kim KR, Park WK. Radiologic features of a tailgut cyst in a neonate: a case report. J Korean Radiol Soc 2008;58:177-180

15 Lee NH. Tailgut cyst in a neonate. J Korean Surg Soc 2010;79: S67-S70

16 Nakagawa M, Hara M, Oshima H, Kitase M, Shibamoto Y. Radiological findings of tailgut cyst in a fetus. J Comput Assist Tomogr 2008;32:210-213

17 Oh JT, Son SW, Kim MJ, Kim L, Kim H, Hwang EH. Tailgut cyst in a neonate. J Pediatr Surg 2000;35:1833-1835

18 Andea AA, Klimstra DS. Adenocarcinoma arising in a tailgut cyst with prominent meningothelial proliferation and thyroid tissue: case report and review of the literature. Virchows Arch 2005; 446:316-321 